\title{
The Androgen Receptor Promotes Cellular Proliferation by Suppression of G-Protein Coupled Estrogen Receptor Signaling in Triple-Negative Breast Cancer
}

\author{
Yan Shen ${ }^{a}$ Fang Yang ${ }^{\text {Wenwen Zhang }}{ }^{a}$ Wei Song ${ }^{b}$ Yuxiu Liuc Xiaoxiang Guan ${ }^{\mathrm{c}}$ \\ aDepartment of Medical Oncology, Jinling Hospital, Medical School of Nanjing University, Nanjing, \\ ${ }^{b}$ Department of Medical Oncology, Jinling Hospital, Southern Medical University, Guangzhou, \\ 'Department of Medical Statistics, Jinling Hospital, Nanjing University, Nanjing, China
}

\section{Key Words}

Triple-negative breast cancer - Androgen receptor • G-protein coupled estrogen receptor • Proliferation

\begin{abstract}
Background/Aims: The targeted therapy for triple-negative breast cancer (TNBC) is still challenging due to poor understanding on its molecular etiology. The androgen receptor (AR) has recently emerged as a prognostic and treatment-predictive marker in breast cancer. However, the role of AR in TNBC remained elusive. Methods: Immunohistochemistry (IHC) was used to detect AR and G-protein coupled estrogen receptor (GPER) expression in tissue microarrays of 165 TNBC patients. Microarray analysis of mRNAs was performed to identify downstream regulators of AR. TNBC cells were cultured with dihydrotestosterone (DHT) alone or in combination with AR knockdown performed with AR shRNA. Cell viability and colony formation were assessed. Western blotting and qRT-PCR were used to examine protein and mRNA expression, respectively. The potential mechanism of AR-mediated GPER suppression was identified by Chromatin immunoprecipitation (ChIP) assay. AR and GPER expressions were also assessed in nude mouse xenografts by IHC. Results: IHC staining showed that the expression of AR was positively associated with tumor size, lymph node metastasis and highgrade tumor in TNBC patients. AR activation triggered by DHT suppressed GPER expression, to promote cell growth of TNBC. G-1, a GPER agonist, inhibited DHT-stimulated proliferation. Further experiments illustrated that AR suppressed GPER activation via binding directly to the promoter of GPER. Moreover, a negative correlation between AR and GPER was observed in MDA-MB-231 tumor cell xenografts and TNBC patient samples. Conclusions: The suppression of GPER via AR may be involved in the positive actions towards the TNBC progression, making it a promising therapeutic target for TNBC treatment.
\end{abstract}

Xiaoxiang Guan

and Yuxiu Liu
Department of Medical Oncology, Jinling Hospital, Medical School of Nanjing University, Department of Medical Statistics, Jinling Hospital, Nanjing University, Nanjing (China); E-Mail xguan@nju.edu.cn/liu_yuxiu@163.com 


\section{Introduction}

Triple-negative breast cancer (TNBC) has been identified as a refractory and aggressive malignancy, comprising 10-17\% of all breast cancer cases [1]. Histologically, it is characterized by the absence of estrogen receptor (ER), progesterone receptor (PR) and epidermal growth factor receptor 2 (HER-2) [2,3], which also results in poor prognosis and high rate of mortality $[4,5]$. Thus, the in-depth elucidation of the molecular events on triple negative breast carcinogenesis could bring about improvements in early diagnosis, prognosis, and treatment of TNBC clinically.

The androgen receptor (AR) is one member of the steroid hormone receptor family, belonging to the superfamily of nuclear receptors. Although multiple biomarkers have been detected in TNBC [6], AR is expressed in more than 70\% of breast cancers [7] and also counts for up to $30 \%$ of TNBC [8]. More recently, emerging researches have implied the critical role of $\mathrm{AR}$ and the associated signaling pathway in the tumorigenesis and development of cancers. AR is a ligand-dependent transcription factor that modulates the expression of targeted genes involved in various physiological and pathological processes [9]. Preclinical studies have also revealed another way of triggering AR activation in a ligand-independent manner through MAPK, JAK/STAT3, NOTCH and PI3K/mTOR/AKT pathways [10-13]. However, to date, the role of AR in TNBC remains uncertain. Recent preclinical studies have presented conflicting data on the biological role of androgens in TNBC and the association of AR expression with prognosis and clinical outcome [14]. For example, one study [15] found that AR expression was positively correlated with increased disease free survival (DFS) in patients with luminal breast cancer, while decreased DFS in TNBC. In contrast, a metaanalysis suggested that AR positivity was associated with prolonged DFS in TNBC, but had no significant impact on overall survival [16]. Another preclinical research detected that tumor proliferation is stimulated by androgens and inhibited by AR antagonists with MDA-MB-453 cell model both in vitro and in vivo [17]. The conflicting action of AR in TNBC appears to be attributed to cross-talk with other complex pathways. Thus, there is an urgent need to investigate the potential effects of AR on TNBC in further studies.

Previous clinical studies have designed to assess the effectiveness of androgen blockade on TNBC. The results of a phase II trial with bicalutamide, an androgen-blocking agent, in AR-positive mTNBC patients were reported. This research supported the active efficacy of hormone-oriented therapy in AR-positive TNBC [14]. Subsequently, another phase II trial of a more-potent AR inhibitor enzalutamide in advanced-stage AR-positive TNBC was reported. Eventually, two complete responses and five partial responses were observed among those 75 evaluable patients with $\mathrm{AR} \geq 10 \%$ [18]. The clinical trials offered the opportunity of ARoriented therapeutic approach for TNBC.

In the present study, we aimed to explore the potential mechanisms of AR in the growth and the development of TNBC. We characterized the expression of AR in TNBC tissues and associated AR expression with clinicopathological data. Then we determined the effects of AR activation triggered by dihydrotestosterone (DHT) on the growth of TNBC in vitro. Furthermore, the role of GPER involved in AR signaling was assessed with TNBC cells, nude mouse xenografts and clinical samples. These researches provided a novel therapeutic approach for TNBC.

\section{Materials and Methods}

\section{Patients and tissue samples}

Tissue microarrays, namely HBreD090Bc01 and HBreD075Bc01, were provided by Outdo Biotech (Shanghai, China). It contained totally 165 cancer cases of triple-negative breast cancer patients, only a single punch for the one patient. Inclusion criteria were female sex, original histological diagnosis of invasive breast carcinoma, negativity for ER, PR and HER2, and availability of clinical pathological data. Experiments were 


\section{Cellular Physiology Cell Physiol Biochem 2017;43:2047-2061 \\ \begin{tabular}{l|l|l} 
and Biochemistry 10.1159/000484187 & $\begin{array}{l}\text { @ } 2017 \text { The Author(s). Published by S. Karger AG, Basel } \\
\text { www.karger.com/cpb }\end{array}$
\end{tabular}}

Shen et al.:Mechanisms of Androgen Receptor Induced TNBC Proliferation

performed in accordance with the Helsinki Declaration and ethics boards of Jinling Hospital. Histological parameters were determined in compliance with the criteria of the World Health Organization.

\section{Immunohistochemistry (IHC) staining and Evaluation}

Slides were deparaffinized in a series of xylenes and ethanols. Heat-mediated antigen retrieval was fulfilled with citrate buffer (BioGenex Laboratories, San Ramon, CA). Antibodies used for Immunohistochemistry staining included: rabbit anti-GPER antibody (BA3433-2, 1:50, Boster), and rabbit anti-AR antibody (ab74272, 1:350, Abcam). Immunostained sections were scanned using a microscope (Aiovert 200, Carl Zeiss). According to previous researches [19, 20], a cut-off of $10 \%$ - those below this being classified as negative and those above being positive - was used in our assessment of AR and GPER expression. All the assessments were done in a blinded manner and determined independently by two senior pathologists.

\section{Microarray analysis}

MDA-MB-231 and Hs578T cells were treated with $100 \mathrm{n}$ M of DHT for 48 hours. The total RNA was extracted from treated or untreated TNBC cells and mRNA was purified from total RNA after removal of rRNA (mRNA-ONLYTM Eukaryotic mRNA Isolation Kit, Epicentre). Then, each sample was amplified and transcribed into fluorescent cRNA along the entire length of the transcripts without 3 ' bias utilizing a random priming method (Arraystar Flash RNA Labeling Kit, Arraystar). The labeled cRNA was then hybridized onto the Microarray v3.0 (ArrayStar). After the washing steps, the Agilent DNA Microarray Scanner (part number G2505C) was used to scan the arrays. Acquired array images were analyzed by Agilent Feature Extraction software (version 11.0.1.1). Quantile normalization and subsequent data processing were performed using The Agilent GeneSpring GX v12.1 software package (Agilent Technologies). The threshold we used to screen dysregulated mRNAs is fold-change $\geq 2.0$ and a $P$-value $\leq 0.05$.

\section{Reagents and Cell culture}

Dihydrotestosterone (DHT, Sigma) was dissolved in ethanol and rel-1-[4-(6-bromo-1, 3-benzodioxol5-yl)-3aR,4S,5, 9bS-tetrahydro-3H-cyclopenta [c]quinolin-8-yl]-ethanone (G-1, Cayman, USA) was solubilized in dimethyl sulfoxide (DMSO). Cells were plated into 6- or 96-well dishes and treated with designated concentrations of DHT or G-1 for indicated time. TNBC cell lines MDA-MB-231 and Hs578T were purchased from the Chinese Academy of Science Committee Type Culture Collection Cell Bank (Shanghai, China). Cells were cultured in RPMI 1640 or DMEM medium (GIBCO, USA) supplemented with $10 \%$ FBS and $1 \%$ penicillin/streptomycin, and 5\% insulin is added for Hs578T cells. Cell cultures were carried out in a humiditied $37^{\circ} \mathrm{C}$ incubator supplied with $5 \% \mathrm{CO}_{2}$.

\section{Plasmids and Transient Transfection}

The sequences of AR short hairpin RNA (shRNA) were 5'-CACCAATGTCAACTCCAGGAT-3', and the sequences of GPER shRNA were 5'-CGAGTTAAAGAGGAGAAGGAA-3'. The two shRNAs, the plasmid AR and negative control were all chemically synthesized by GenePharma (Shanghai, China). Cells $\left(1 \times 10^{6}\right.$ cells/ well) were plated in 6-well plates $24 \mathrm{~h}$ prior to transfection and transfected using Lipofectamine 2000 reagent (Invitrogen), according to the manufacturer's protocol. After $6 \mathrm{~h}$ of transfection, we replaced the transfection medium with the normal one. Cells were harvested for testing when they occupied $80 \%$ of the plate.

\section{Cell viability assay}

A total of $1 \times 10^{4}$ cells per well were seeded into 96-well plates in regular growth medium. Cells were washed once they had attached and then incubated in medium containing specific concentrations of DHT, G-1 or transfected with required plasmids for $48 \mathrm{~h}$. Following addition of $20 \mu \mathrm{L}$ of $0.5 \mathrm{mg} / \mathrm{mL}$ dimethylthiazoldiphenyltetrazoliumbromide (MTT, Sigma-Aldrich) solution to each well, the medium was replaced with $200 \mu \mathrm{L} \mathrm{DMSO}$ after $4 \mathrm{~h}$ and vortexed for $10 \mathrm{~min}$. Absorbance was measured with a microplate reader (BIO-RAD, USA) at a test wavelength of $490 \mathrm{~nm}$. Each experiment was performed in triplicate.

Colony formation assay

Cells were seeded in six-well plates at a density of 500 cells per well for approximately $24 \mathrm{~h}$ under standard conditions. After specific treatments, the cells then were exposed to DHT alone or in combination 
with G-1. After nearly two weeks of incubation, the colonies were fixed with methanol, stained with $0.5 \%$ crystal violet in absolute ethanol, and colonies with $\geq 50$ cells were counted under a dissection microscope. These experiments were repeated at least three times.

\section{Western blot analysis}

Total protein was extracted using RIPA buffer supplemented with protease and phosphatase inhibitors according to the manufacturer's protocol. Approximately $20 \mu \mathrm{g}$ protein was loaded per lane and separated on a sodium dodecylsulfate-polyacrylamide gel and blotted onto nitrocellulose. Blots were blocked with $5 \%$ dry milk in tris-buffered saline/0.1\% tween-20 and incubated overnight with a diluted solution of primary antibody at $4{ }^{\circ} \mathrm{C}$, and then with incubation with a horseradish peroxidase-conjugated secondary antibody (1:5000) for $2 \mathrm{~h}$. Antibodies used for western blot included: rabbit anti-GPER antibody (BA34332, 1:500, Boster), and rabbit anti-AR antibody (ab74272, 1:200, Abcam). Bands were normalized to GAPDH expression. Results from at least two separate experiments were analyzed.

\section{Quantitative real-time PCR}

Total RNA was extracted from cells with the RNAiso Plus reagent (Takara, Japan) according to the manufacturer's protocol. Total cDNA was synthesized in a reaction mix containing 2 mg of total RNA and PrimeScript RT reagent Kit with gDNA Eraser (Takara, Japan). qRT-PCR was implemented with Step One (TM) sequence detection system (Applied Biosystems Inc, Milan, Italy) using validated primers and SYBR Premix Ex Taq II (Takara, Japan), following the manufacturer's instructions. The cycle number at threshold $(\mathrm{Ct}$ ) was used to quantify the transcript levels of genes of interest. Primer pairs were as follows: AR, forward 5'-GGGCGAAGTAGAGCATCCT-3' and reverse 5'-GACGACCAGATGGCTGTCATT-3'; GPER, forward 5' $5^{\prime}$-AGTCGGATGTGAGGTTCAG-3' and reverse $5^{\prime}$-TCTGTGTGAGGAGTGCAAG-3'; GAPDH, forward 5'-TGTTGCCATCAATGACCCCTT-3' and reverse 5'-CTCCACGACGTACTCAGCG-3'. Assays were performed in triplicate and the results were normalized for GAPDH expression and then calculated as fold induction of RNA expression.

\section{Chromatin immunoprecipitation (ChIP) assay}

The ChIP assay was performed using Pierce Agarose ChIP Kit (Thermo) according to the manufacturer's instructions. In brief, 70\% confluent MDAMB-231 cells were treated with ethanol or $100 \mathrm{n}$ M DHT for $2 \mathrm{~h}$ and then fixed in 1\% formaldehyde for $15 \mathrm{~min}$. Centrifugation for $5 \mathrm{~min}$, and sonicate the lysate to shear the cross-linked DNA to an average length of 100-1000 bp. Remove the insoluble material by centrifugation. The immuno-cleared chromatin was immunoprecipitated with anti-AR (Ab74272, 1:200, abcam) or control IgG antibody overnight at $4^{\circ} \mathrm{C}$. Reverse the cross-links by proteinase $\mathrm{K}$ in ChIP Elution buffer for $1.5 \mathrm{~h}$ at $65^{\circ} \mathrm{C} .1 \mu \mathrm{l}$ of each of the purified DNA was used to determine the concentration. Each sample was used as template for PCR with specific primers. 10 primer sets were designed for the GPER promoter region. Primer sequences were designed for about every $300 \mathrm{bp}$ of each promoter set shown in Table 1, as the sequences amplified

Table 1. The following sequences of primers were designed for GPER promoter in ChIP analysis. 10 primer sets of about $300 \mathrm{bp}$ per primer were synthesized for the GPER promoter region. The sequences amplified by primer set 1 is the fastest from the transcription start site

\begin{tabular}{|c|c|c|}
\hline \multirow[t]{2}{*}{ No. } & \multicolumn{2}{|c|}{ Sequences of primers for GPER promoter ( $5^{\prime}$ to $3^{\prime}$ ) } \\
\hline & Forward & Reverse \\
\hline 1 & CAGCTGACTAAAGTGTGGCT & CCTAATTTTGTTAAATGAACTCCAAGG \\
\hline 2 & AAAGAAAATAAACGTTAAGTTGAGGG & АTTCTAAAAGTACTGAATAACСTTCAC \\
\hline 3 & AACTGACACAGAGGCAGTGAT & GGCGAAACCCTGTCTCTAC \\
\hline 4 & ATGTTACCCAGGCTGGTCTC & TTAGTAGAGATGGGGTTTTACCATG \\
\hline 5 & AAATATAAAACATTAGCCAGGCGTGG & CAAGCCCAGCAGAGAATTACCT \\
\hline 6 & GGCACCTGTCCTAGGCACTGTA & TСССАТСАСССАССССGТ \\
\hline 7 & CССТCСCСACTGGCGGCA & GCAATGCTGTTTGTAACCTAGGAAGAAAC \\
\hline 8 & GGTTACAAACAGCATTGCAAAAAC & GGGAAAATGCATTTCAGCAGC \\
\hline 9 & СТСТССТСАСАAАTGCCG & GTGTCTGTAGAGTCTGGGT \\
\hline 10 & CACGCGGGACTGTGCACGGT & GTCTCTGCACCGTGCAGCTTTC \\
\hline
\end{tabular}


Shen et al.:Mechanisms of Androgen Receptor Induced TNBC Proliferation

by primer sets 1 is the fastest from the transcription start site. PCR products were separated on $2 \%$ agarose gels, stained with ethidium bromide and photo each.

\section{Statistical analysis}

Statistical analysis was performed with SPSS Statistics 19.0 (SPSS Inc.) and GraphPad Prism 6. Data were analyzed using one-way ANOVA or a Student's t-test. Data are presented as means \pm SD of three independent experiments. The chi-square test was used for IHC data. The correlation between AR and GPER expression level was estimated using Pearson's correlation analysis. A $P$ value of $<0.05$ was considered statistically significant. ${ }^{*} P$ $<0.05,{ }^{*} P<0.001$.
Table 2. Correlation of AR expression with patient's clinical and pathological characteristics ${ }^{\text {a }}$ Chi-square test. AR, Androgen receptor. ${ }^{*} P<0.05$

\begin{tabular}{|c|c|c|c|c|c|c|}
\hline \multirow[t]{2}{*}{ Variables } & \multirow[t]{2}{*}{ Cases } & \multicolumn{2}{|c|}{$\begin{array}{l}\text { AR low }^{\text {ln=106) }}\end{array}$} & \multicolumn{2}{|c|}{$\begin{array}{l}\text { AR }{ }^{\text {high }} \\
(n=59)\end{array}$} & \multirow[t]{2}{*}{$P$ value } \\
\hline & & & $\%$ & No. & $\%$ & \\
\hline \multicolumn{6}{|l|}{ Age(years) } & \multirow[t]{3}{*}{0.514} \\
\hline$\leq 50$ & 91 & 56 & 52.8 & 35 & 59.3 & \\
\hline$>50$ & 74 & 50 & 47.2 & 24 & 40.7 & \\
\hline \multicolumn{6}{|c|}{ Tumour size $(\mathrm{cm})$} & \multirow[t]{3}{*}{$0.006^{*}$} \\
\hline$\leq 2$ & 68 & 52 & 49.1 & 16 & 27.1 & \\
\hline$>2$ & 97 & 54 & 50.9 & 43 & 72.9 & \\
\hline \multicolumn{6}{|c|}{ Lymph node status } & \multirow[t]{3}{*}{$0.019^{*}$} \\
\hline Negative & 131 & 90 & 84.9 & 41 & 69.5 & \\
\hline Positive & 34 & 16 & 15.1 & 18 & 30.5 & \\
\hline \multicolumn{6}{|l|}{ TNM stage } & \multirow[t]{4}{*}{0.821} \\
\hline I & 7 & 5 & 4.7 & 2 & 3.4 & \\
\hline II & 130 & 82 & 77.4 & 48 & 81.4 & \\
\hline III & 28 & 19 & 17.9 & 9 & 15.3 & \\
\hline \multicolumn{6}{|c|}{ Pathological stage } & \multirow[t]{4}{*}{$0.011^{*}$} \\
\hline I & 66 & 51 & 48.1 & 15 & 25.4 & \\
\hline II & 92 & 50 & 47.2 & 42 & 71.2 & \\
\hline III & 7 & 5 & 4.7 & 2 & 3.4 & \\
\hline
\end{tabular}

\section{Results}

Activation of AR via DHT promotes in vitro viability of TNBC

Recent studies have found that the androgen receptor (AR) is present in approximately $60-70 \%$ of all breast cancers, and in up to about $30 \%$ of patients with triple-negative breast cancer (TNBC) [21], making it an attractive therapeutic target and, likely, a prognostic marker. In this present study, we analyzed the expression of AR in a tissue microarray of 165 TNBC tumors by IHC. Representative low-expression and high-expression AR immunostainings of TNBC samples were shown in Fig. 1A. Of the 165 tumor tissues, 59 cases (35.8\%) and 106 cases (64.2\%) expressed AR at high and low levels, respectively (Table 2). Importantly, the expression level of AR is significantly $(P<0.05)$ positively associated with tumor size, highgrade tumors and lymph node metastasis (Table 2).

Thus, we investigated the roles of AR in the motility of TNBC cells. In line with our published data, which demonstrated that AR inhibitor exhibits antiproliferative effect in mesenchymal stem-like (MSL) TNBC cells [22], we observed the positive effect of DHT, a well-known AR agonist, on cellular viability of two AR-positive MSL TNBC cells, namely MDA-MB-231 and Hs578T. We assessed the positive action of increasing concentrations of DHT at 0.1 to $100 \mathrm{n} \mathrm{M}$ on MDA-MB-231 and Hs578T cells by MTT assay. A dose-dependent enhancement of cell proliferation was observed, and it was significantly induced at the highest concentration of $100 \mathrm{n} \mathrm{M} \mathrm{(Fig.} \mathrm{1B).} \mathrm{Furthermore,} \mathrm{we} \mathrm{attempted} \mathrm{to} \mathrm{evaluate} \mathrm{the} \mathrm{active}$ effects of DHT on cellular viability in different time durations. MDA-MB-231 and Hs578T cells were treated with DHT at the concentration of $100 \mathrm{n} \mathrm{M}$ and samples were collected at different time points $(24,48$, or $72 \mathrm{~h})$. As shown in Fig. 1C, DHT treatment enhanced cellular proliferation at different time points in the two cell lines mentioned above. Moreover, we determined the expression of AR in MDA-MB-231 and Hs578T, treated with DHT by western blot analysis. As shown in Fig. 1D, DHT treatment resulted in a significant increase of AR protein expression in the two TNBC cells. Additionally, qRT-PCR analysis showed that DHT treatment noticeably upregulated AR transcript levels in both MDA-MB-231 and Hs578T cells (Fig. 1E). Furthermore, we knocked down AR in the presence of DHT and found that AR deficiency significantly blocked DHT-induced cell proliferation in the two TNBC cells (Fig. 1F). Taken together, these data manifest that AR activation via DHT promotes cell proliferation of TNBC. 


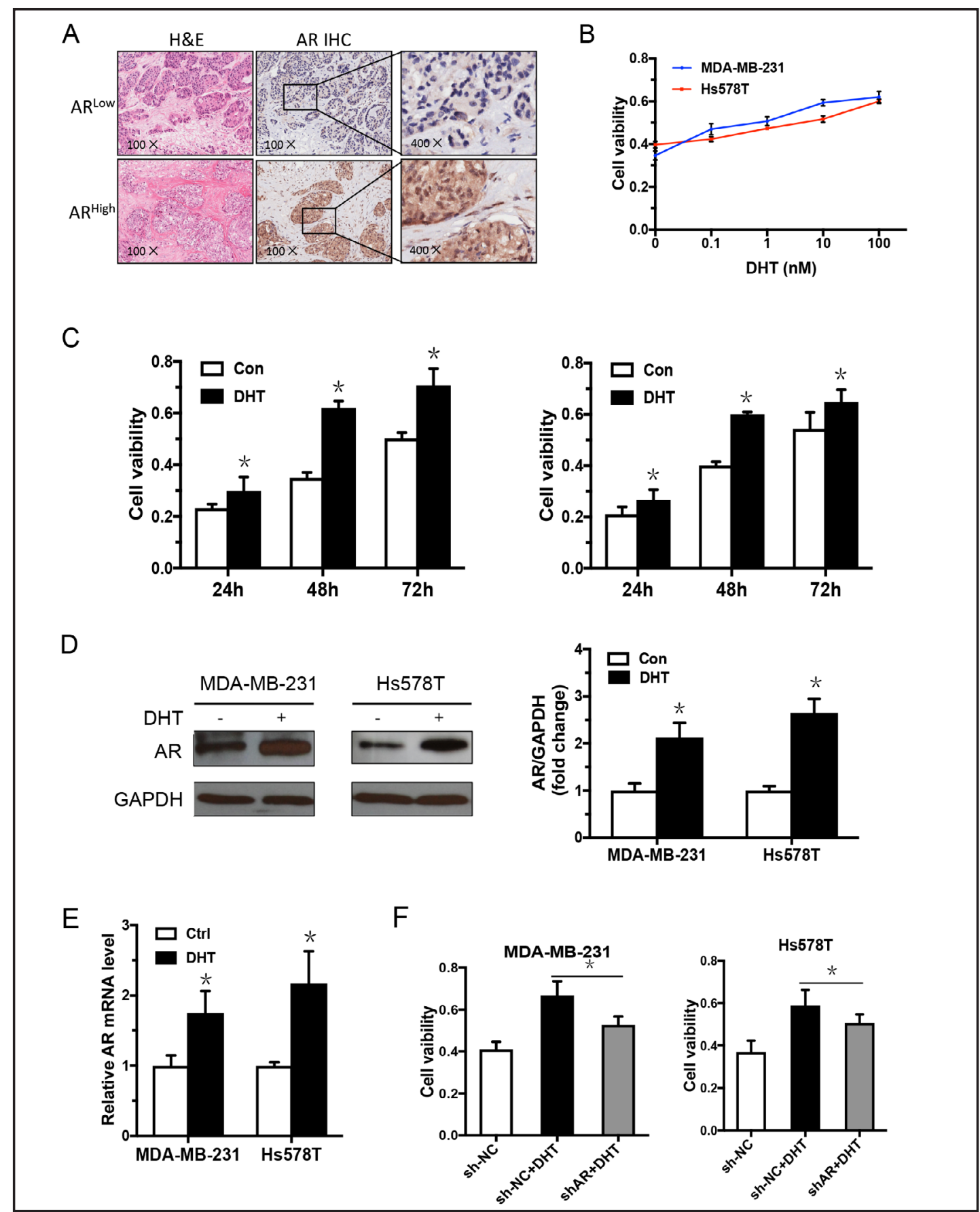

Fig. 1. Immunohistochemical analysis of AR in TNBC samples and the effect of DHT on cell viability. (A) IHC analysis of AR low-expression (the upper panels) and high-expression (the lower panels) in representative TNBC tissue samples. Tissue slices were Hematoxylin and eosin (H\&E) stained (left). The middle slices were stained for AR (magnification, $\times 100$ ). Panels on the right show higher magnification (magnification, $\times 400$ ). (B) Cell viability of MDA-MB-231 and Hs578T cells was measured by MTT assay following treatment with DHT at indicated concentrations for $48 \mathrm{~h}$. (C) Cell viability was measured by MTT assay following treatment with $100 \mathrm{n}$ M DHT for 24, 48, or $72 \mathrm{~h}$. The expression of AR in MDA-MB-231 and Hs578T cells treated with $100 \mathrm{n}$ M DHT or vehicle for $48 \mathrm{~h}$ was determined by Western blotting (D) or qRT-qPCR (E), respectively, and normalized to GAPDH expression. (F) Cell viability was measured by MTT assay following treatment with $100 \mathrm{n}$ M DHT and/or transfection with shAR for $48 \mathrm{~h}$ in MDA-MB-231 and Hs578T cells, respectively. Data represented the mean of three independent experiments. ${ }^{*} \mathrm{P}<0.05$. 


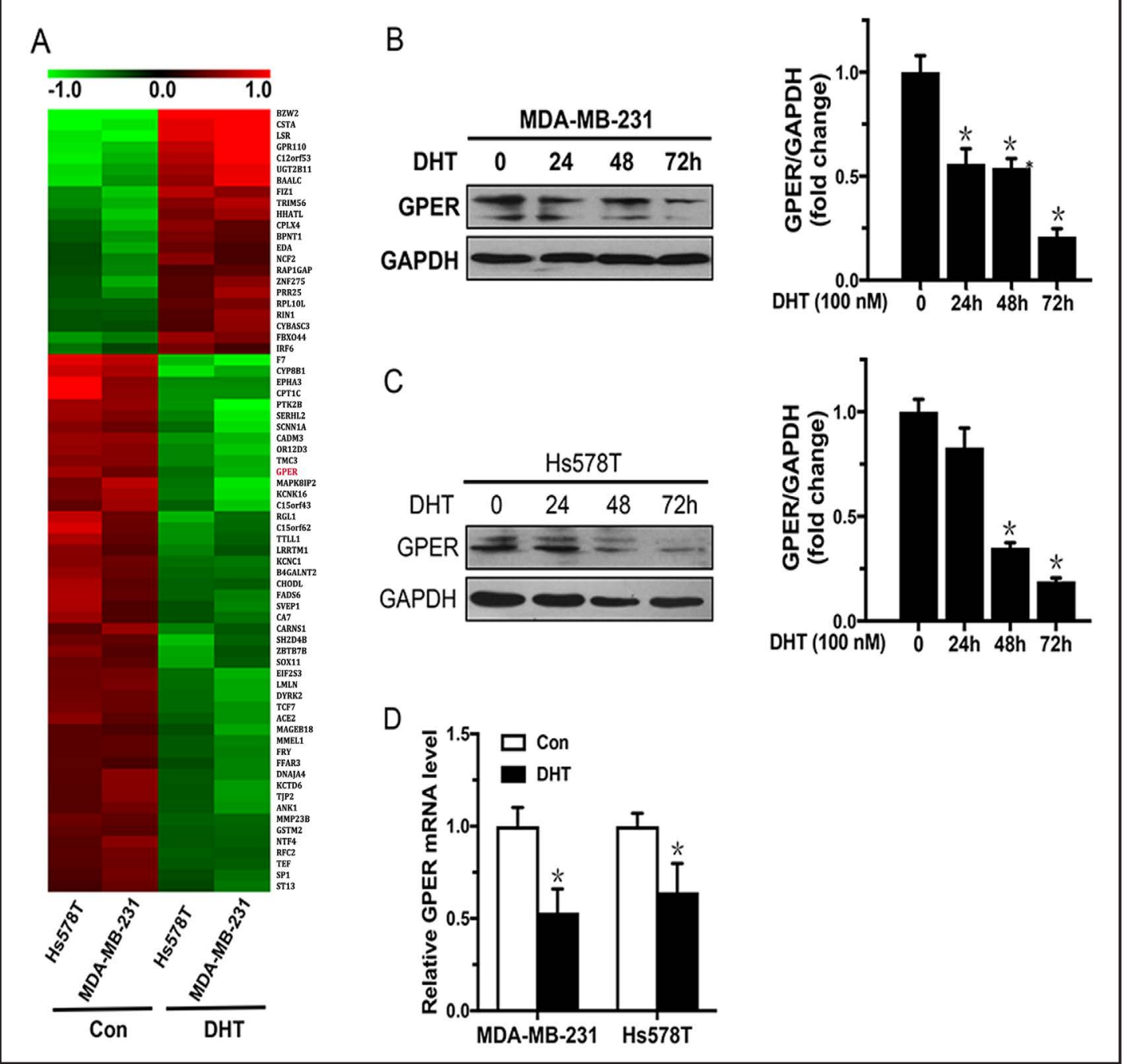

Fig. 2. GPER was downregulated by DHT in TNBC cell lines. (A) Heat map showing DHT-mediated gene expression. GPER is shown in red. Microarray analysis was utilized to profile gene expression in MDAMB-231 and Hs578T cells followed by either $100 \mathrm{n}$ M DHT treatment or vehicle for $48 \mathrm{~h}$. The heat maps represent fold changes in the expression of DHT-regulated genes. Each row represents one gene, and each column corresponds to one expression microarray. The expression of GPER was detected by Western blotting following the treatment of vehicle or $100 \mathrm{n}$ M DHT for 24, 48, or $72 \mathrm{~h}$ in MDA-MB-231 (B) and Hs578T cells (C), and normalized to GAPDH expression. (D) Relative mRNA expression of GPER in MDA-MB-231 and Hs578T cells was detected by qRT-qPCR in MDA-MB-231 and Hs578T cells followed by either $100 \mathrm{n}$ M DHT treatment or vehicle for $48 \mathrm{~h}$. Data represented the mean of three independent experiments. ${ }^{*} \mathrm{P}<0.05$.

\section{GPER expression is inhibited by DHT}

To identify the targeted genes regulated by AR and involved in the process of ARstimulated proliferation, we treated MDA-MB-231 and Hs578T cells with vehicle or DHT at $100 \mathrm{n} \mathrm{M}$ for $48 \mathrm{~h}$. We then conducted microarray analysis to compare mRNA expression levels between DHT treated and untreated cells, and found 23 remarkably upregulated and 48 remarkably downregulated mRNAs in both two cell lines in DHT treated group compared with control group (Fig. 2A). The seven-transmembrane G protein-coupled estrogen receptor (GPER, also known as GPR30), a member of G protein-coupled receptor (GPCR), has been reported to be activated by estrogen to modulate the progression of various hormone-responsive tumors including ER positive breast cancer [23]. Intriguingly, recent 


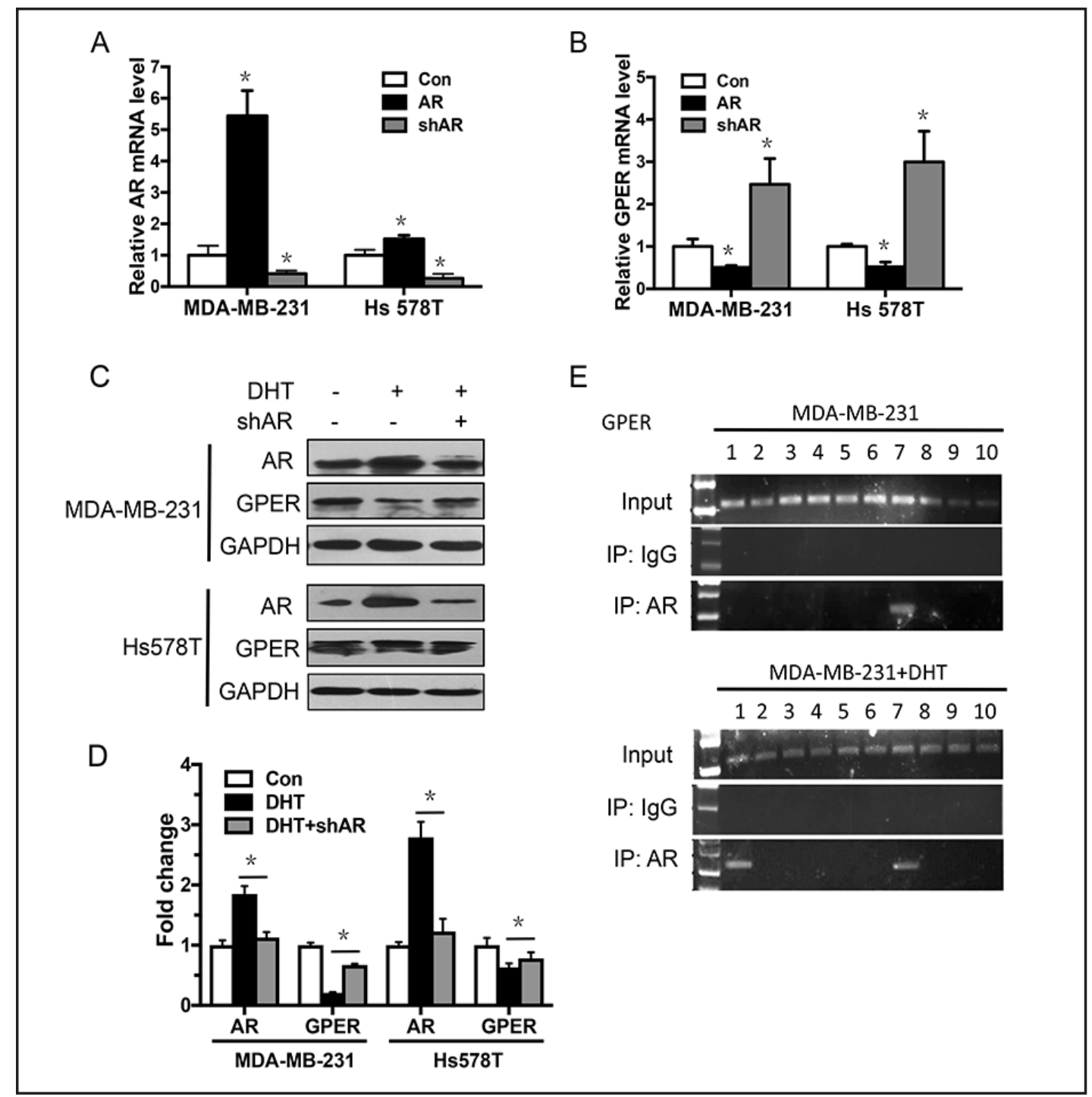

Fig. 3. DHT suppressed GPER expression through the activation of AR by binding to its promoter. AR (A) and GPER mRNA levels (B) were determined by qRT-qPCR in MDA-MB-231 and Hs578T cells following treatment with control vector or AR vector or transfection with shAR for $48 \mathrm{~h}$. (C) The protein expression of AR and GPER was determined by Western blotting in MDA-MB-231 and Hs578T cells following treatment with DHT and/or transfection with shAR for $48 \mathrm{~h}$. (D) Densitometric analysis of AR and GPER is shown below, normalized to GAPDH expression. (E) ChIP assay was performed using AR antibody in MDA-MB231cells. AR immunoprecipitated fraction was subjected to PCR amplification with the ten primers sets of GPER promoter. Amplification was observed with primer sets 7 (lane 7) in vehicle-treated controls and primer sets 1 and 7 (lane 1,7) after DHT treatment. Data represented the mean of three independent experiments. ${ }^{*} \mathrm{P}<0.05$.

studies indicated that GPER was also greatly expressed in TNBC cell lines and patient tissues $[24,25]$. Therefore it is worthy to investigate the role of GPER-signaling on AR-mediated proliferation of TNBC in absence of ER. Meanwhile, we have predicted the interaction of AR with mRNAs among the dysregulated mRNAs more than 2 fold-change, using the String prediction algorithm (http://string-db.org). Interestingly, GPER, one of the downregulated mRNAs, which has a relatively large fold change, is predicted to interact with AR, indicating a strong possibility as a downstream regulator of AR. 


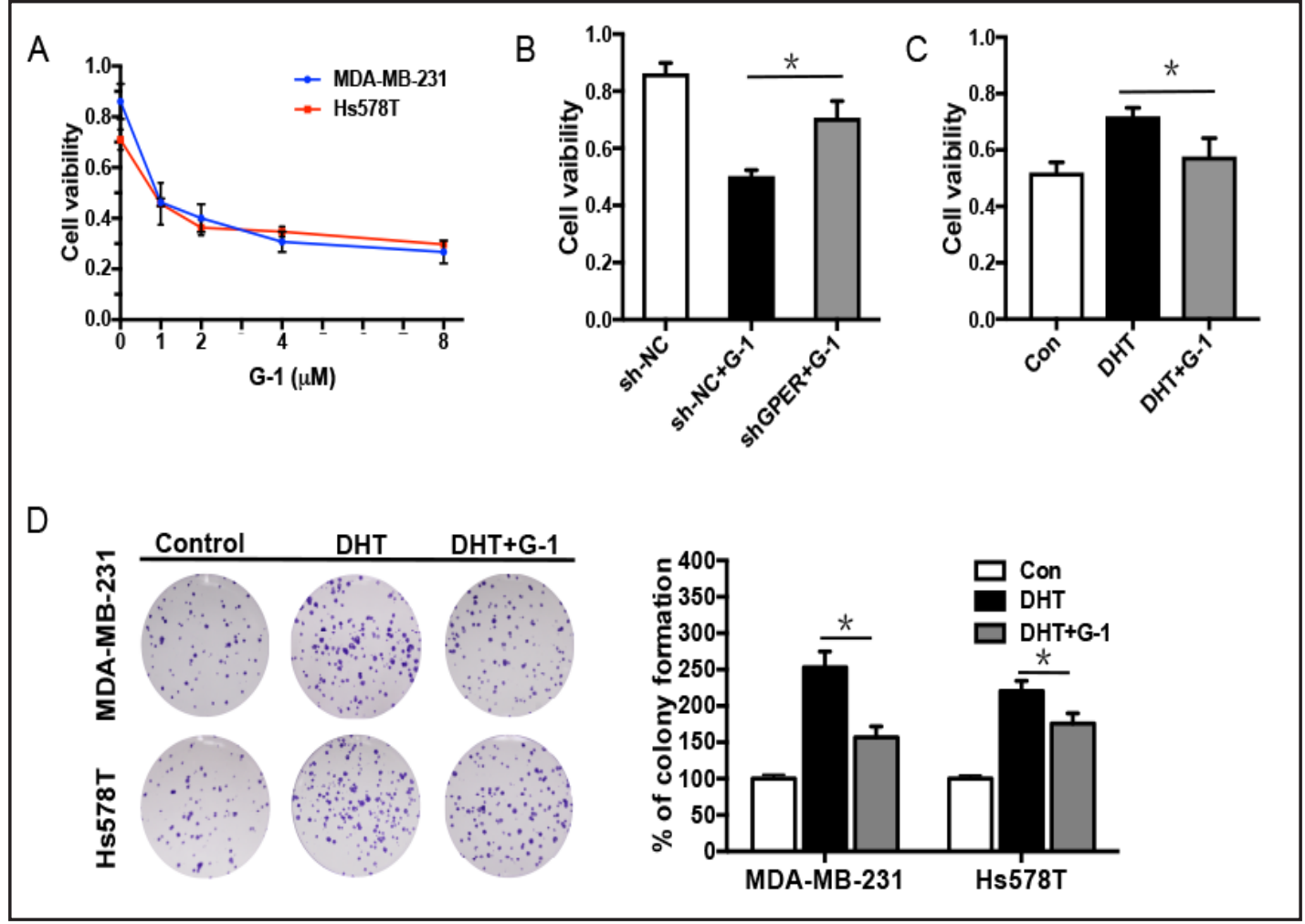

Fig. 4. The activation of GPER attenuates the action of DHT on stimulating tumor growth. (A) Cell viability of MDA-MB-231 and Hs578T cells was measured by MTT assay following treatment with G-1 at indicated concentrations for $48 \mathrm{~h}$. (B) Cell viability of MDA-MB-231 cells was measured by MTT assay following treatment with G-1 and/or transfection with shGPER for $48 \mathrm{~h}$. (C) Cell viability of MDA-MB-231 cells was measured by MTT assay following treatment with DHT and/or G-1 for 48 h. (D) MDA-MB-231 and Hs578T cells were treated with DHT and G-1 alone or in combination for 48h, then cell proliferation was determined by clone formation assay. Data represented the mean of three independent experiments. ${ }^{*} \mathrm{P}<0.05$.

To confirm the microarray results, we treated MDA-MB-231 and Hs578T cells with DHT and measured the expression of GPER at various time points by western blot analysis. As shown in Fig. 2B and Fig. 2C, DHT treatment induced a decrease of GPER protein expression in different time durations $(24,48$, or $72 \mathrm{~h})$ in the two cells. Meanwhile, we detected GPER mRNA expression upon DHT treatment in the two cells by qRT-PCR analysis, and observed a robust decrease in the transcript levels of GPER compared with the control group (Fig. 2D, $P<0.05$ ). Collectively, these data provide the evidence that DHT negatively regulates GPER expression in TNBC cells.
Table 3. Correlation analysis to evaluate the association between AR, GPER expression in 165 breast cancer tissues. AR, Androgen receptor; GPER, G-protein coupled estrogen receptor. ${ }^{* *} \mathrm{P}<0.001$

\begin{tabular}{|c|c|c|}
\hline & \multirow{2}{*}{\multicolumn{2}{|c|}{$\begin{array}{l}\text { Tumor microarray }(\mathrm{n}=165) \\
\text { ARlow }\end{array}$}} \\
\hline & & \\
\hline GPERlow $(n=57)$ & 26 & 31 \\
\hline $\operatorname{GPER}^{\text {high }}(\mathrm{n}=108)$ & 80 & 28 \\
\hline r & -0.282 & \\
\hline$P$ & 0.0003 & \\
\hline
\end{tabular}

Activation of AR triggered by DHT inhibits GPER expression by binding to its promoter

To investigate the regulatory actions of AR on GPER expression, qRT-PCR analysis of GPER expression in the AR-transfected or AR-silenced cells was performed. A significant decrease of GPER mRNA levels was detected in the AR-transfected group of MDA-MB-231 and Hs578T cells, while an increase in the AR-silenced group (Fig. 3A and Fig. 3B). To further assess the role of AR in DHT-mediated GPER expression, we knocked down AR in the 


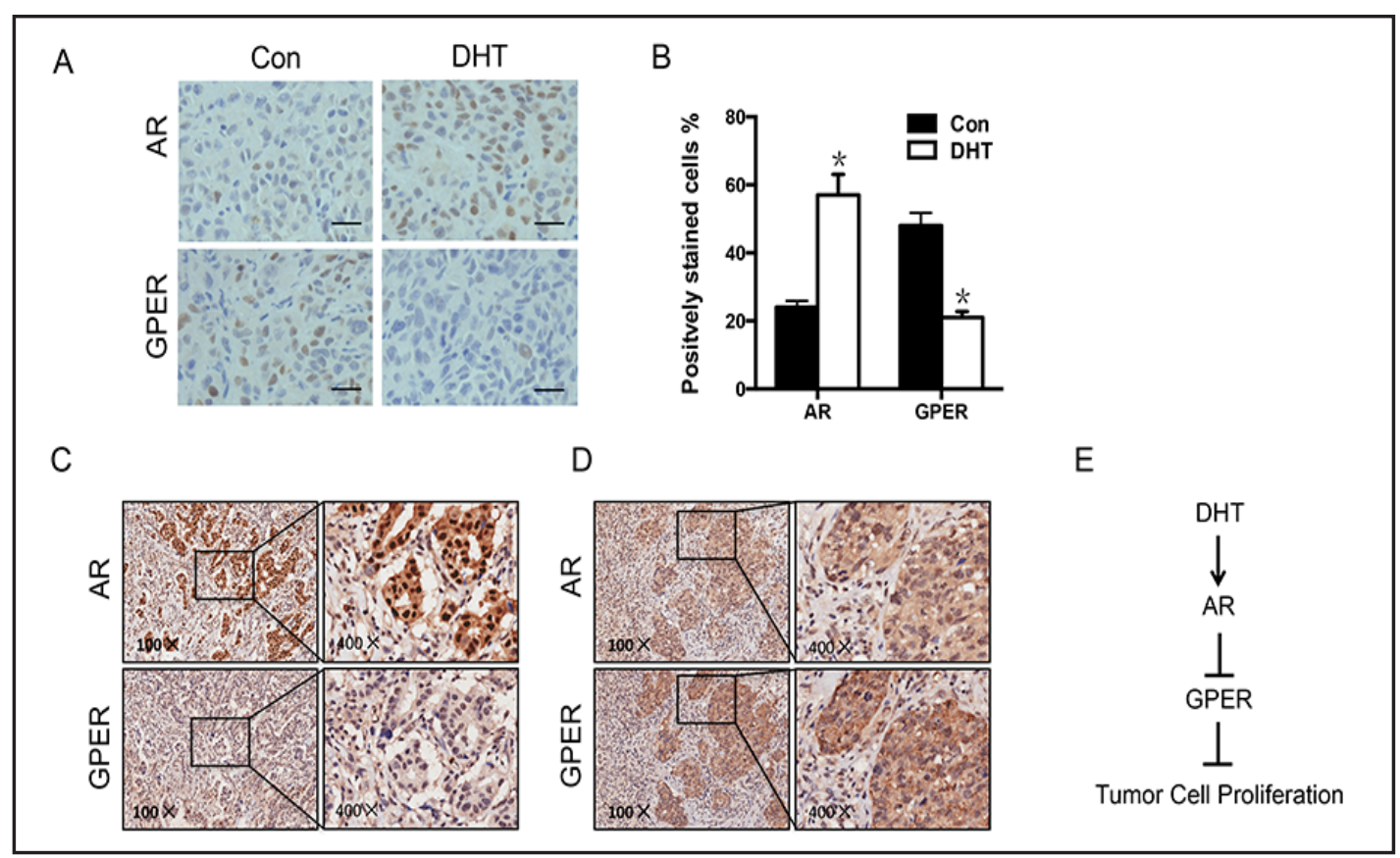

Fig. 5. A negative correlation between AR and GPER expression in axenograft tumors and TNBC patients. (A) The tumor sections were subjected to histological analysis by H\&E staining and IHC staining of indicated proteins. Photomicrographs depict representative AR, GPER staining (400x). Scale bars represent 50 $\mu \mathrm{m}$. (B) AR, GPER protein expression in MDA-MB-231 xenografts were quantified in IHC. Representative immunohistochemical staining of AR high- and GPER low-expression (C) or AR low- and GPER highexpression (D) in breast cancer patient tissues. (E) A model showing a DHT-AR-GPER axis signaling pathway involved in tumor cell proliferation. Our data indicate that DHT treatment induces the activation of AR, which inhibits GPER and potentially other factors, eventually leading to cell proliferation. Data represented the mean of three independent experiments. ${ }^{*} \mathrm{P}<0.05$.

presence of DHT to examine whether AR affected DHT-mediated GPER expression. Western blot assay showed that AR deficiency significantly attenuated GPER suppression mediated by DHT (Fig. 3C and Fig. 3D), suggesting the critical role of AR in mediating DHT-suppressed GPER signaling. Taken together, we propose that AR activation leads to the suppression of GPER.

To investigate the mechanisms of AR-induced proliferation in TNBC cells and whether AR directly interacted with GPER, we performed ChIP assay in MDA-MB-231 cells to immunoprecipitate cell chromatin with an anti-AR antibody and amplify the site located within the GPER promoter region. Our results illustrated that AR was strongly recruited at the promoter of GPER, located at -1509 to - $1240 \mathrm{bp}$ upstream of transcription start site (Fig. 3E, lane 7). We observed that DHT administration enabled AR to bind to the region of GPER promoter located at -3000 to -2706 bp (Fig. 3E, lane 1), indicating that DHT-induced AR activation enhanced the regulation of AR on the transcription of GPER. In brief, our results suggested that AR negatively regulated the expression of GPER through directly binding to its promoter as a transcription repressor. Moreover, DHT administration facilitated this procedure and made the binding more effectively.

The activation of GPER attenuates the stimulation effects of DHT on cellular proliferation

To evaluate the effects of GPER on DHT-induced cell growth, increasing concentrations of G-1, a well-known GPER agonist, were used to investigate the function of GPER in TNBC cells by MTT assay. Dose-dependent inhibition of cell growth was observed, and the treatment of G-1 at $1 \mu \mathrm{M}$ concentration resulted in a viability decrease of $45 \%$ and $35 \%$ in MDA-MB-231 
and Hs578T cells, respectively (Fig. 4A). In line with the previous studies [26-28], the dose of $1 \mu \mathrm{M}$ G-1 was selected for further in vitro studies. Furthermore, we conducted knockdown assays in MDA-MB-231 cells to assess whether the inhibitory effects of G-1 depended on GPER activation. As shown in Fig. 4B, the silencing of GPER by shRNA in the presence of G-1 abolished the inhibitory effects of G-1 on cell viability of MDA-MB-231 cells. These data revealed that G-1 suppressed cellular proliferation of TNBC through the activation of GPER. Therefore, we hypothesized that the activation of GPER through G-1 could attenuate DHTstimulated proliferation of TNBC cells. Then MTT assays were performed to verify the role of GPER on DHT-induced motility of TNBC cells, it showed that addition of G-1 in the presence of DHT treatment blocked cell viability by 19\% compared with DHT-alone group (Fig. 4C). Consistently, colony formation assays exhibited that DHT treatment enhanced the ability of colony formation, while the activation of GPER by G-1 attenuated DHT-simulated colony formation by about $21 \%$ and $15.3 \%$, respectively, in MDA-MB-231 and Hs578T cells (Fig. $4 \mathrm{D})$. Collectively, these results demonstrate that GPER is a key downstream regulator in the DHT-mediated process of cellular proliferation in TNBC cells.

AR-mediated GPER suppression is manifested in xenograft tumors and TNBC patients

Since we have demonstrated that GPER was involved in AR mediated oncogenic processes in TNBC cell lines, there was little direct evidence for the correlation of GPER and AR in vivo. Our previous studies have revealed that DHT treatment significantly accelerated the in vivo growth of MDA-MB-231 xenograft tumors in comparison with the control treatment [22]. In the present study, to assess the effects of DHT treatment on AR and GPER levels in the primary tumor xenografts, IHC staining analysis was conducted on the harvested xenogrft tumors obtained from our previous experiment as mentioned. Of note, an increase of AR expression and a reduction of GPER levels were found in paraffin-embedded tumor tissues obtained from DHT stimulated mice with respect to mice treated with vehicle (Fig. 5A and Fig. 5B, $P<0.05)$. These data were consistent with the in vitro experimental model that DHT stimulated motility of TNBC cells and suppressed GPER expression through the activation of AR. Taken together, these data indicate that GPER, which could be suppressed by AR activation, is involved in the tumor growth of TNBC prompted by DHT.

To further provide clinical evidence for the role of AR and GPER in TNBC, we used an IHC staining analysis to assess the expression of AR and GPER in specimens from 165 TNBC patients using a tissue microarray. Representative staining is shown in Fig. 5C. 108 cases (65.5\%) and 57 cases (34.5\%) expressed GPER at high and low levels among the 165 tumor tissues, respectively (Table 3). A significant negative correlation between AR and GPER expression was identified using the TNBC tissue-array ( $\mathrm{r}=-0.282, P<0.001)$ (Table 3). Taken together, our observations support the negative correlation between AR and GPER expression in tumor xenografts and TNBC patients.

\section{Discussion}

It is reported that AR plays a critical role in the progression of breast cancer cells, while its role in TNBC remains largely unknown. This present study showed that the expression of AR was positively associated with tumor size, lymph node metastasis and high-grade tumor in TNBC patients. Furthermore, we demonstrated that the AR agonist DHT promoted cellular proliferation of TNBC. In particular, we found out that DHT significantly inhibited expression of GPER through the activation of AR, which was mechanically confirmed by ChIP assay suggesting the directly binding of AR to the GPER promoter. Meanwhile, the over expression of GPER induced by the selective GPER agonist G-1 abolished AR-stimulated proliferative changes in TNBC cells. Our published study demonstrated that DHT treatment significantly promoted tumorigenesis in MDA-MB-231 xenografts mice. In the present study, we further observed the high expression of AR while low expression of GPER in xenograft tumors with DHT treatment. Consistent with our in vitro and in vivo studies, clinical data manifested that the expression of AR is negatively correlated with GPER expression while positively 
associated with tumor size, high-grade tumors, lymph node metastasis in 165 cases of TNBC patients.

AR, which can be activated by DHT, is widely expressed in various cancer types, involving in malignant progression. A recent review summarized conflicting data of preclinical researches on the complex role of AR in TNBC [29]. In our study, we detected that AR was highly expressed in about $35.8 \%$ of TNBC patients. Importantly, the expression level of AR was positively associated with tumor size, high-grade tumors and lymph node metastasis, indicating the association of AR expression with poor diagnosis. Our data were further supported by Jiang et al. [15]. who showed that AR expression was associated with decreased DFS in TNBC, but were not in agreement with a recent study, which indicated that AR expression was associated with favorable prognosis such as smaller tumor size, lower grade, and overall survival, particularly in the luminal breast cancer subtypes [30]. Nevertheless, emerging evidence implied intratumoral heterogeneity as a considerable issue that might influence the suitability of core biopsy. Diffuse staining, that is multiple punches taken for the one patient, may help evaluate the impact of intratumoral heterogeneity on the determination of AR and GPER expression in our experiment. Thus, further studies using a larger sample size with diffuse staining are needed to confirm these data.

As a member of nuclear receptors, AR mediates the biological process of cell cycle regulation and cell proliferation. In a ligand-independent manner, AR is located in the cytoplasm and forms a complex with heat shock protein. While in a ligand-dependent manner AR binds with DHT, AR homodimer will then translocate to nuclear and interact with androgen responsive elements to regulate transcription of downstream genes [31]. Here, we reported that the activation of AR via DHT exhibits the proliferative effects on TNBC cell lines. Actually, our results are consistent with the previous observations that AR drove tumor progression in some subtypes of TNBC models [32,33], as well as in AR+/ER- breast cancer cells [34, 35]. Meanwhile, some ER-positive BC cell lines, such as MCF-7 and MDAMB-453, were growth-stimulated by androgens and inhibited by anti-androgens $[17,36]$. In particular, AR expression has also been shown to influence neoadjuvant chemotherapy efficacy in the GeparTrio phase III study [37]. Nevertheless there exist some controversial studies suggesting that AR signaling antagonized the growth stimulatory effect of ER signaling in ER-positive breast cancer [33,38]. Additionally, Tilley and colleagues demonstrated that two steroidal androgens inhibited proliferation in AR-positive breast cancer cell lines [39]. Owing to multiple players involved in TNBC, the complex role of AR remains elusive. Our results confirmed a proliferative role of AR in TNBC cells. Thus, further study is needed to confirm these data.

G-protein coupled estrogen receptor (GPER), a member of G-protein coupled receptor (GPCR), has been reported to modulate the progression of a variety of cancer types such as ovarian, endometrial, and breast cancers $[14,40]$. Previous researches showed that GPER regulated the activities of epidermal growth factor receptors (EGFRs), mitogen-activated protein kinase (MAPK) and phosphoinositide 3-kinase (PI3K) signaling pathways [41, 42]. In this study, we conducted a microarray to explore the upstream regulator of AR. Interestingly, we observed a significant reduction of GPER level after DHT treatments. This is further confirmed that DHT suppressed GPER expression through the activation of AR. Further in vitro researches illustrated that activation of GPER through G-1 attenuated DHT-induced proliferation. Coincidentally, our current data are consistent with another study indicating that G-1 inhibited the in vivo growth and invasive potential of TNBC [43]. Consistent to the in vitro studies, the negative correlation of AR and GPER was confirmed in patient tissues and nude mouse xenografts. To date this report is the first time to illustrate the molecular events of GPER involved in AR-mediated pathway and elucidate the negative correlation between AR and GPER in TNBC patients.

As AR acts as transcription factor, we determined whether AR directly interacts with the region of GPER promoter. The direct interaction was confirmed by ChIP analysis, showing that AR occupied the promoter region of GPER. The transcription of GPER is negatively modulated through direct binding of AR to its promoter. We propose that AR occupies the 
promoter region of GPER and competitively blocks the binding of the positive regulators to GPER promoter, ultimately leading to the down-regulation of GPER. Furthermore, DHT enhanced the inhibitory effects of AR on the transcription of GPER. Collectively, these results suggest that androgen-activated AR may suppress GPER expression, eliciting tumor stimulative actions on TNBC.

\section{Conclusion}

We report for the first time, that activation of AR via DHT may promote proliferation of TNBC through down regulation of GPER. Furthermore, our clinical data demonstrate that the expression of AR is negatively associated with GPER while positively associated with tumor size, lymph node metastasis and high-grade tumor in TNBC patients. This paper provides the rationale and data for the potential target therapy of AR and GPER in TNBC in future. Thus, further studies are needed to confirm our current findings and develop a potential strategy to treat TNBC clinically.

\section{Acknowledgements}

This study was supported by National Natural Science Foundation of China (No. 81470357; No.81473066) and a Foundation for Clinical Medicine Science and Technology Special Project of the Jiangsu Province, China (No. BL2014071).

\section{Disclosure Statement}

All authors declare no Disclosure Statement.

\section{References}

1 Reis-Filho JS, Tutt AN: Triple negative tumours: a critical review. Histopathology 2008;52:108-118.

-2 de Ruijter TC, Veeck J, de Hoon JP, van Engeland M, Tjan-Heijnen VC: Characteristics of triple-negative breast cancer. J Cancer Res Clin Oncol 2011;137:183-192.

- 3 Yang N, Zhou TC, Lei XX, Wang C, Yan M, Wang ZF, Liu W, Wang J, Ming KH, Wang BC, Xu BL, Liu Q: Inhibition of Sonic Hedgehog Signaling Pathway by Thiazole Antibiotic Thiostrepton Attenuates the CD44+/CD24Stem-Like Population and Sphere-Forming Capacity in Triple-Negative Breast Cancer. Cell Physiol Biochem 2016;38:1157-1170.

4 Bosch A, Eroles P, Zaragoza R, Vina JR, Lluch A: Triple-negative breast cancer: molecular features, pathogenesis, treatment and current lines of research. Cancer Treat Rev 2010;36:206-215.

-5 Dent R, Trudeau M, Pritchard KI, Hanna WM, Kahn HK, Sawka CA, Lickley LA, Rawlinson E, Sun P, Narod SA: Triple-negative breast cancer: clinical features and patterns of recurrence. Clin Cancer Res 2007;13:44294434.

6 Chen X, Wu J, Huang H, Ding Q, Liu X, Chen L, Zha X, Liang M, He J, Zhu Q, Wang S, Xia T: Comparative Profiling of Triple-Negative Breast Carcinomas Tissue Glycoproteome by Sequential Purification of Glycoproteins and Stable Isotope Labeling. Cell Physiol Biochem 2016;38:110-121.

7 McNamara KM, Yoda T, Takagi K, Miki Y, Suzuki T, Sasano H: Androgen receptor in triple negative breast cancer. J Steroid Biochem Mol Biol 2013;133:66-76.

-8 McNamara KM, Moore NL, Hickey TE, Sasano H, Tilley WD: Complexities of androgen receptor signalling in breast cancer. Endocr Relat Cancer 2014;21:T161-181.

-9 Mangelsdorf DJ, Thummel C, Beato M, Herrlich P, Schutz G, Umesono K, Blumberg B, Kastner P, Mark M, Chambon P, Evans RM: The nuclear receptor superfamily: the second decade. Cell 1995;83:835-839. 


\section{Cellular Physiology Cell Physiol Biochem 2017;43:2047-2061

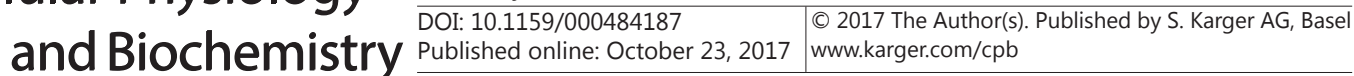

Shen et al.:Mechanisms of Androgen Receptor Induced TNBC Proliferation

10 Cuenca-Lopez MD, Montero JC, Morales JC, Prat A, Pandiella A, Ocana A: Phospho-kinase profile of triple negative breast cancer and androgen receptor signaling. BMC Cancer 2014;14:302.

11 Garay JP, Karakas B, Abukhdeir AM, Cosgrove DP, Gustin JP, Higgins MJ, Konishi H, Konishi Y, Lauring J, Mohseni M, Wang GM, Jelovac D, Weeraratna A, Sherman Baust CA, Morin PJ, Toubaji A, Meeker A, De Marzo AM, Lewis G, Subhawong A, Argani P, Park BH: The growth response to androgen receptor signaling in ERalpha-negative human breast cells is dependent on p21 and mediated by MAPK activation. Breast Cancer Res 2012;14:R27.

-12 Tarulli GA, Butler LM, Tilley WD, Hickey TE: Bringing androgens up a NOTCH in breast cancer. Endocr Relat Cancer 2014;21:T183-202.

13 Ueda T, Bruchovsky N, Sadar MD: Activation of the androgen receptor N-terminal domain by interleukin- 6 via MAPK and STAT3 signal transduction pathways. J Biol Chem 2002;277:7076-7085.

-14 Gucalp A, Tolaney S, Isakoff SJ, Ingle JN, Liu MC, Carey LA, Blackwell K, Rugo H, Nabell L, Forero A, Stearns V, Doane AS, Danso M, Moynahan ME, Momen LF, Gonzalez JM, Akhtar A, Giri DD, Patil S, Feigin KN, Hudis CA, Traina TA, Translational Breast Cancer Research C: Phase II trial of bicalutamide in patients with androgen receptor-positive, estrogen receptor-negative metastatic Breast Cancer. Clin Cancer Res 2013;19:55055512.

15 Jiang HS, Kuang XY, Sun WL, Xu Y, Zheng YZ, Liu YR, Lang GT, Qiao F, Hu X, Shao ZM: Androgen receptor expression predicts different clinical outcomes for breast cancer patients stratified by hormone receptor status. Oncotarget 2016;7:41285-41293.

16 Wang C, Pan B, Zhu H, Zhou Y, Mao F, Lin Y, Xu Q, Sun Q: Prognostic value of androgen receptor in triple negative breast cancer: A meta-analysis. Oncotarget 2016;7:46482-46491.

17 Cochrane DR, Bernales S, Jacobsen BM, Cittelly DM, Howe EN, D’Amato NC, Spoelstra NS, Edgerton SM, Jean A, Guerrero J, Gomez F, Medicherla S, Alfaro IE, McCullagh E, Jedlicka P, Torkko KC, Thor AD, Elias $\mathrm{AD}$, Protter AA, Richer JK: Role of the androgen receptor in breast cancer and preclinical analysis of enzalutamide. Breast Cancer Res 2014;16:R7.

18 Traina TA MK, Yardley DA, O’Shaughnessy J, Cortes J, Awada A, Kelly CM, Trudeau ME, Schmid P, Gianni L: Results from a phase 2 study of enzalutamide (ENZA), an androgen receptor (AR) inhibitor, in advanced AR+ triple-negative breast cancer (TNBC). Journal of Clinical Oncology 2015;S33.

19 Bonnefoi H, Grellety T, Tredan O, Saghatchian M, Dalenc F, Mailliez A, L'Haridon T, Cottu P, AbadieLacourtoisie S, You B, Mousseau M, Dauba J, Del Piano F, Desmoulins I, Coussy F, Madranges N, Grenier J, Bidard FC, Proudhon C, MacGrogan G, Orsini C, Pulido M, Goncalves A: A phase II trial of abiraterone acetate plus prednisone in patients with triple-negative androgen receptor positive locally advanced or metastatic breast cancer (UCBG 12-1). Ann Oncol 2016;27:812-818.

20 Zhou X, Wang S, Wang Z, Feng X, Liu P, Lv XB, Li F, Yu FX, Sun Y, Yuan H, Zhu H, Xiong Y, Lei QY, Guan KL: Estrogen regulates Hippo signaling via GPER in breast cancer. J Clin Invest 2015;125:2123-2135.

21 Niemeier LA, Dabbs DJ, Beriwal S, Striebel JM, Bhargava R: Androgen receptor in breast cancer: expression in estrogen receptor-positive tumors and in estrogen receptor-negative tumors with apocrine differentiation. Mod Pathol 2010;23:205-212.

-22 Zhu A, Li Y, Song W, Xu Y, Yang F, Zhang W, Yin Y, Guan X: Antiproliferative Effect of Androgen Receptor Inhibition in Mesenchymal Stem-Like Triple-Negative Breast Cancer. Cell Physiol Biochem 2016;38:10031014.

-23 Ariazi EA, Brailoiu E, Yerrum S, Shupp HA, Slifker MJ, Cunliffe HE, Black MA, Donato AL, Arterburn JB, Oprea TI, Prossnitz ER, Dun NJ, Jordan VC: The G protein-coupled receptor GPR30 inhibits proliferation of estrogen receptor-positive breast cancer cells. Cancer Res 2010;70:1184-1194.

24 Steiman J, Peralta EA, Louis S, Kamel O: Biology of the estrogen receptor, GPR30, in triple negative breast cancer. Am J Surg 2013;206:698-703.

25 Yu T, Liu M, Luo H, Wu C, Tang X, Tang S, Hu P, Yan Y, Wang Z, Tu G: GPER mediates enhanced cell viability and motility via non-genomic signaling induced by 17 beta-estradiol in triple-negative breast cancer cells. J Steroid Biochem Mol Biol 2014;143:392-403.

-26 Madeo A, Maggiolini M: Nuclear alternate estrogen receptor GPR30 mediates 17beta-estradiol-induced gene expression and migration in breast cancer-associated fibroblasts. Cancer Res 2010;70:6036-6046.

-27 Pandey DP, Lappano R, Albanito L, Madeo A, Maggiolini M, Picard D: Estrogenic GPR30 signalling induces proliferation and migration of breast cancer cells through CTGF. EMBO J 2009;28:523-532. 


\section{Cellular Physiology Cell Physiol Biochem 2017;43:2047-2061

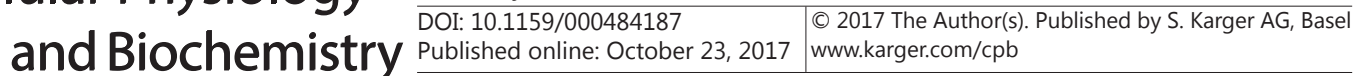

Shen et al.:Mechanisms of Androgen Receptor Induced TNBC Proliferation

28 Wei W, Chen ZJ, Zhang KS, Yang XL, Wu YM, Chen XH, Huang HB, Liu HL, Cai SH, Du J, Wang HS: The activation of G protein-coupled receptor 30 (GPR30) inhibits proliferation of estrogen receptor-negative breast cancer cells in vitro and in vivo. Cell Death Dis 2014;5:e1428.

29 Narayanan R, Dalton JT: Androgen Receptor: A Complex Therapeutic Target for Breast Cancer. Cancers (Basel) 2016;8:

-30 Aleskandarany MA, Abduljabbar R, Ashankyty I, Elmouna A, Jerjees D, Ali S, Buluwela L, Diez-Rodriguez M, Caldas C, Green AR, Ellis IO, Rakha EA: Prognostic significance of androgen receptor expression in invasive breast cancer: transcriptomic and protein expression analysis. Breast Cancer Res Treat 2016;159:215-227.

-31 Pietri E, Conteduca V, Andreis D, Massa I, Melegari E, Sarti S, Cecconetto L, Schirone A, Bravaccini S, Serra P, Fedeli A, Maltoni R, Amadori D, De Giorgi U, Rocca A: Androgen receptor signaling pathways as a target for breast cancer treatment. Endocr Relat Cancer 2016;23:R485-498.

-32 Barton VN, D’Amato NC, Gordon MA, Lind HT, Spoelstra NS, Babbs BL, Heinz RE, Elias A, Jedlicka P, Jacobsen BM, Richer JK: Multiple molecular subtypes of triple-negative breast cancer critically rely on androgen receptor and respond to enzalutamide in vivo. Mol Cancer Ther 2015;14:769-778.

33 Chia K, O’Brien M, Brown M, Lim E: Targeting the androgen receptor in breast cancer. Curr Oncol Rep 2015;17:4.

34 Naderi A, Chia KM, Liu J: Synergy between inhibitors of androgen receptor and MEK has therapeutic implications in estrogen receptor-negative breast cancer. Breast Cancer Res 2011;13:R36.

-35 Ni M, Chen Y, Lim E, Wimberly H, Bailey ST, Imai Y, Rimm DL, Liu XS, Brown M: Targeting androgen receptor in estrogen receptor-negative breast cancer. Cancer Cell 2011;20:119-131.

-36 Birrell SN, Bentel JM, Hickey TE, Ricciardelli C, Weger MA, Horsfall DJ, Tilley WD: Androgens induce divergent proliferative responses in human breast cancer cell lines. J Steroid Biochem Mol Biol 1995;52:459-467.

-37 Loibl S, Muller BM, von Minckwitz G, Schwabe M, Roller M, Darb-Esfahani S, Ataseven B, du Bois A, FisslerEckhoff A, Gerber B, Kulmer U, Alles JU, Mehta K, Denkert C: Androgen receptor expression in primary breast cancer and its predictive and prognostic value in patients treated with neoadjuvant chemotherapy. Breast Cancer Res Treat 2011;130:477-487.

-38 Lim E, Ni M, Cao S, Hazra A, Tamimi RM, Brown M: Importance of Breast Cancer Subtype in the Development of Androgen Receptor Directed Therapy. Curr Breast Cancer Rep 2014;6:71-78.

-39 Cops EJ, Bianco-Miotto T, Moore NL, Clarke CL, Birrell SN, Butler LM, Tilley WD: Antiproliferative actions of the synthetic androgen, mibolerone, in breast cancer cells are mediated by both androgen and progesterone receptors. J Steroid Biochem Mol Biol 2008;110:236-243.

40 Wang C, Lv X, He C, Hua G, Tsai MY, Davis JS: The G-protein-coupled estrogen receptor agonist G-1 suppresses proliferation of ovarian cancer cells by blocking tubulin polymerization. Cell Death Dis 2013;4:e869.

41 Ge C, Yu M, Zhang C: G protein-coupled receptor 30 mediates estrogen-induced proliferation of primordial germ cells via EGFR/Akt/beta-catenin signaling pathway. Endocrinology 2012;153:3504-3516.

42 Luo LJ, Liu F, Lin ZK, Xie YF, Xu JL, Tong QC, Shu R: Genistein regulates the IL-1 beta induced activation of MAPKs in human periodontal ligament cells through G protein-coupled receptor 30. Arch Biochem Biophys 2012;522:9-16.

-43 Chen ZJ, Wei W, Jiang GM, Liu H, Wei WD, Yang X, Wu YM, Liu H, Wong CK, Du J, Wang HS: Activation of GPER suppresses epithelial mesenchymal transition of triple negative breast cancer cells via NF-kappaB signals. Mol Oncol 2016;10:775-788. 